\title{
Automated Detection and Extraction of Brain Tumor from MRI Images
}

\author{
Neha Tirpude \\ Assistant Professor \\ SBJITMR, Nagpur
}

\author{
Rashmi Welekar \\ Assistant Professor \\ SRCOEM, Nagpur
}

\begin{abstract}
Image segmentation algorithms and techniques find its applications in a wide number of domains. Segmentation of brain tumor and overall internal structure of the brain is one of the main applications in the field of medical imaging.

Magnetic resonance imaging (MRI) technique is one of the many imaging modalities that are available to scan and capture the internal soft tissue structures of the body. In this paper, proposed technique has been given to extract the tumor portion, successfully demarcate the tumor boundary, locate the tumor with a bounding circle and to diagnose whether the tumor is present or absent. A fuzzy clustering-based technique is proposed which helps to study \& analyze the intricate structure of the brain, hence can be used as a visual analysis and a study tool.
\end{abstract}

\section{General Terms}

Image Processing, Pattern Recognition

\section{Keywords}

MRI, Magnetic resonance imaging, image segmentation, fuzzy clustering, thresholding

\section{INTRODUCTION}

Magnetic resonance imaging (MRI) technique is one of the many available imaging modalities like CT-Scan, Mammography, and X-Ray. It provides visual details about the anatomy and the overall structure of the brain. An MRI scan can be used to study the supply of blood inside the brain. Hence MRI technology becomes an important tool for detecting abnormality, tracking the progress or growth of the disease and for diagnosis too. Processing digital images by means of a digital computer comprises digital image processing domain [1].

Brain tumors are caused due to abnormal, uncontrolled growth of cells. Primary tumors are those that originate in the brain. Secondary tumors are those that originate in some other part of the body, finally reaching the brain through the process of metastasis.

The symptoms of brain tumors include headache, nausea, vomiting, personality and behavioral changes, memory loss, sensory disturbance, weakness, numbness [2].

\subsection{MRI Scan}

MRI is a fairly new technique that has been used since the beginning of the 1980s. The MRI scanner uses magnetic and radio waves to create pictures of tissues, organs and other structures within the body, which can then be viewed on a computer. There is no exposure to X-rays or any other damaging forms of radiation in MRI.
The pictures produced by an MRI scan are better in displaying fine details and therefore are of higher diagnostic quality when compared to more frequently used X-ray scanners for example.

\subsection{Utility of MRI Images}

Using an MRI scanner, it is possible to make pictures of almost all the tissue in the body. The tissue that has the least hydrogen atoms (such as bones) turns out dark, while the tissue that has many hydrogen atoms (such as fatty tissue) looks much brighter.

By changing the timing of the radio wave pulses, it is possible to gain information about the different types of tissues that are present.

An MRI of the brain and spinal cord can be performed to look at different abnormalities, as it can provide clear images of these structures in spite of being surrounded by bone tissue. Changes within the tissues of brain, whether subtle or gross, can aid in diagnosis and to determine the treatment.

For example, an MRI of the brain can be done to look for the changes associated with bleeding or when the brain has been starved of oxygen after a stroke. It can be used to investigate a traumatic brain injury, and also help diagnose developmental abnormalities. It can be used to characterize tumors (benign or malignant abnormal growths) and check their progression, for example if they have changed in size and whether there has been spread to nearby tissues. Within the heart, an MRI scan can give very detailed imaging of the thickness and size of the chamber walls. Damage occurring to the heart tissue after a heart attack or in association with valvular heart disease can also be assessed.

Other structures like the large blood vessels within the surrounding tissue can also be examined, for example to check for the buildup of plaques, which can predispose the patient to having a heart attack in the future. Congenital cardiovascular conditions can more accurately be assessed with MRI, along with changes to the heart which have occurred as a consequence of these conditions. MRI is the imaging modality of choice for assessing joint problems. This is because joints are typically made up of a number of very different tissues, for example muscle, bone and ligaments. MRI is very effective at giving clear images of these individual tissues. The method can also sometimes be used to monitor other parts of the body such as the liver, kidneys, spleen and breasts.

\section{LITERATURE REVIEW}

Dina A. Dahab et al. have used a modified image segmentation algorithm and have also proposed a probabilistic neural network algorithm which has given a very fast classification and the accuracy rate of classification was also 
found to be higher than the conventional neural network system [3]. M. Sinha and K. Mathur have proposed a system to improve the detection rate and they also have integrated it with ontology-based technique dealing with classes and subclasses [4].

A variation of the fuzzy clustering technique was also used with a gradient vector flow snake model in order to segment the brain image [5]. N. Noreen et al. have integrated image segmented by fuzzy c-means and the image transformed by Wavelet and have later enhanced the edges using Kirch's mask [6].

S. Mohsin et al. have focused on efficient and faster technique of skull stripping as a major portion of the preprocessing phase; they have combined erosion step along with selection of area of interest [7].

Along with thresholding operation, watershed segmentation was also used to mark the region of the thresholded tumor [8]. P. Vasuda and S. Satheesh have proposed a fuzzy-based clustering in order to detect tumors and also have compared the conventional and modified clustering algorithm [9].

A. Dasgupta has given a technique that has overcome the drawback of fuzzy clustering with noisy images [10]. M. Rakesh and T. Ravi have used an integration of color-based segmentation along with K-means clustering and histogram-clustering to separate timorous object from other components [11].

I. Soesanti et al. have integrated spatial information into the membership function of the fuzzy c-means clustering algorithm which is based on fuzzy logic and have also used a neighboring effect to obtain the statistics of cluster distribution.

\section{THE PROPOSED APPROACH \\ 3.1 MRI Image Acquisition}

1. Data collection: The input MRI images have been collected in DICOM (Digital Imaging \& Communications in Medicine) and JPEG (Joint Pictures Expert Group) format; Courtesy: Galaxy Diagnostics, Nagpur and Government Medical College \& Hospital, Nagpur. All images are of type T2. The images have been captured on machines Philips Achieva and G.E. Signa 1.5 Tesla. The various imaging artefacts that appear on the MRI film are "1.5 Tesla, machine name, ET, relaxation time TR, longitudinal TE, name of the body part under scan, image format, scanning direction of slices(axial, sagittal, coronal), name of the institute (e.g. GMCH), name of the patient, age, gender, patient ID, date $\&$ time of capturing scan and image size.

2. Image format Conversion: Majority of the images collected was in DICOM format, hence they have been exported into a more usable format i.e. the JPEG format. This conversion was done using the MicroDicom tool.

3. Removal of film artifacts: Any MRI image film consists of a number of film artefacts such as the machine details, patient details \& imaging parameters like TR, TE. Since these artefacts may interfere as 'white' content, they need to be removed.
4. Size normalization: The images have been acquired from different MRI machines; hence they are variable in size. All images have been size normalized to be of size $256 * 256$ pixels.

\subsection{Image Enhancement Phase}

1. Read the input brain MRI image.

2. Remove the color components by changing the image mode to gray-scale.

3. Make the edges in the image clear and crisp by sharpening the components.

4. Enhance the quality of the image by applying a median filter.

5. Plot the histogram to study and analyze the intensity distribution of the pixels.

\subsection{MRI Image Segmentation Phase}

1. Perform a simple binary thresholding on the MRI image.

2. Find all the connected components

3. Convert the image to get the colored connected components. This gives an idea about how and which regions are connected to one another.

4. Global Thresholding: In our previous work, we have implemented two of the standard global thresholding techniques- basic global thresholding and Otsu's thresholding. Results in [16] have shown that for MRI images, both these techniques yielded poor output and have failed to give an overall good segmentation; basic global thresholding [17] made the tumor disappear completely whereas the standard Otsu's [18] method distorted the shape of all the components of the brain MRI image, as the grey-level distribution was found to be homogenous and was not distinct enough to create distinct peaks in the histogram mode.

5. Fuzzy Clustering: Input to the algorithm is the data array ' $\mathrm{D}$ ' and number of clusters ' $\mathrm{N}$ '.

a. Browse the file path; choose the image and read the input MRI image(JPEG format)

b. Convert the image to double to increase the range of pixel values

c. Convert the image to color i.e. RGB image

d. Perform contrast enhancement and display the image

e. Initialize a data array consisting of all rows of input image I

f. From the GUI, enter the number of clusters $\mathrm{N}$ to be formed and pass this value to the fuzzy clustering module

g. For $\mathrm{N}=2$, perform the following: 
1) Create a vector that gives the maximum membership grade for every pixel from vector $\mathrm{U}$

2) Initialize ind1 vector such that it contains only the positions index whenever any element of vector ' $m a x$ ' equals any element of row 1 of vector $U$

3) Repeat similarly for ind2 vector by comparing elements of vector ' $m a x$ ' and row 2 of vector $U$

4) Assign a membership value to ind1 and ind 2 classes.

Combine the membership grades and class values; map the respective pixels to form the final clustered image.

\subsection{Post-Processing Phase}

\section{a) Tumor Extraction and Skull Stripping}

A square-shaped structuring element was used as an identity matrix. Two consecutive erosion operations were applied since a single operation didn't give the expected result. A counter-balancing operation consisting of two consecutive dilations was performed to form the 'base' image.

\section{b) Tumor Demarcation}

Calculate the black and white outline from the perimeter computation of the base image. Set the output image $\mathrm{O}$ same as the original input image. Combine the original image with the perimeter image. Set the color of the demarcation outline. Display the image $\mathrm{O}$ with the tumor demarcated clearly.

c) Tumor With Bounding-Circle:

a. From our base image, calculate the image statistics about the connected white region.

b. The statistics provide an estimated region radius.

c. The region property internally computes parameters like area, centroid and bounding-box statistics.

d. $\mathrm{N}$ is the number of objects or elements detected in stats

e. FOR $\mathrm{i}=1$ to $\mathrm{N}$ do the following

1) Measure the required property i.e. .BoundingBox

2) Compute the radius of the bounding box.

3) Plot the bounding circle over the input brain MRI image.

d) Tumor Detection:

a. From the base image, calculate the total number of pixels with intensity $=255$.

b. Compute the total white region percentage from the complete $256 * 256$ sized image. c. The components that remain after morphological operations, are either tumorous or are normal brain tissue remains.

After analyzing the percentage of high intensity pixels over a large number of images, the threshold value was set to be 0.5 . It was analyzed whether the bits and pieces that remain after all processing are distinctly located at different places. If the percentage of high intensity pixels is found to be greater than 0.50 and the processed remains are not discretely located, then that image is declared tumorous, else the image is declared as non-tumorous.

\section{EXPERIMENTAL RESULTS}

The proposed approach was tested on a database of 80 images. The recorded entities were as follows: the image name, size of tumor, percentage of white region, execution time and the detection decision (' $\mathrm{P}$ ' for tumor present and ' $\mathrm{A}$ ' for tumor absent). The Positive predictive value (PPV) for this proposed system was found to be $86 \%$ and negative predictive value (NPV) was found to be $73.07 \%$.

Some of the table entries are given below in Table 1.

\section{COMPARATIVE ANALYSIS}

The method proposed in this paper was compared with the existing method presented by M. Othman et al. [19] The comparative analysis is presented in Table 2:

\section{Table 2. Comparison with existing method}

\begin{tabular}{|c|c|c|}
\hline $\begin{array}{c}\text { Comparison of } \\
\text { performance }\end{array}$ & $\begin{array}{c}\text { Image } \\
\text { set } \\
\text { used }\end{array}$ & Accuracy \\
\hline M. Othman et al. & 35 & $73.00 \%$ \\
\hline Proposed method & 80 & $77.50 \%$ \\
\hline
\end{tabular}

Fig 1: Flowchart of proposed work

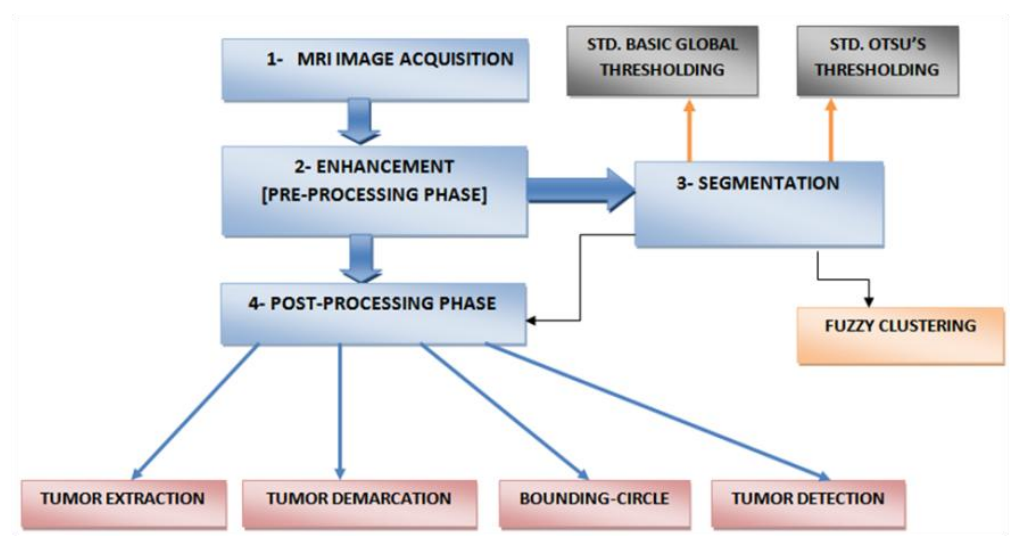


Table 1. Table of selective results

\begin{tabular}{|c|c|c|c|c|c|}
\hline \multirow{2}{*}{$\begin{array}{l}\text { Image } \\
\text { name }\end{array}$} & \multicolumn{2}{|c|}{ Size of tumor } & \multirow{2}{*}{$\begin{array}{l}\text { Percentage of White } \\
\text { region }\end{array}$} & \multirow{2}{*}{$\begin{array}{l}\text { Execution } \\
\text { time }\end{array}$} & \multirow{2}{*}{$\begin{array}{c}\text { Detection decision } \\
\text { Present-P/ Absent- } \\
\text { A }\end{array}$} \\
\hline & $\begin{array}{c}\text { In } \\
\text { pixels }\end{array}$ & $\begin{array}{c}\text { In } \\
\text { inches/area }\end{array}$ & & & \\
\hline 3 & 624 & 0.067 & 0.952 & 3.283 & $\mathbf{P}$ \\
\hline 5 & 17882 & 1.94 & 27.28 & 2.585 & $\mathbf{P}$ \\
\hline $\mathbf{N 2}$ & 288 & 0.031 & 0.439 & 8.106 & $\mathbf{A}$ \\
\hline 30 & 1154 & 0.125 & 1.760 & 4.318 & $\mathbf{P}$ \\
\hline N15 & 112 & 0.012 & 0.170 & 4.230 & $\mathbf{A}$ \\
\hline
\end{tabular}

\section{Case 2: Tumor Image}

Case 1: Non-tumorous image

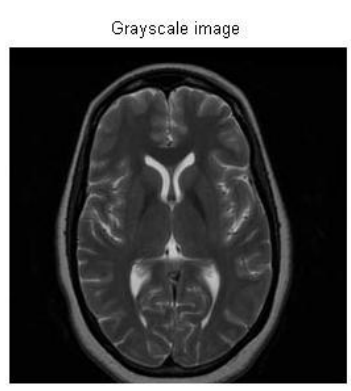

Figure 2: Non-tumorous Image

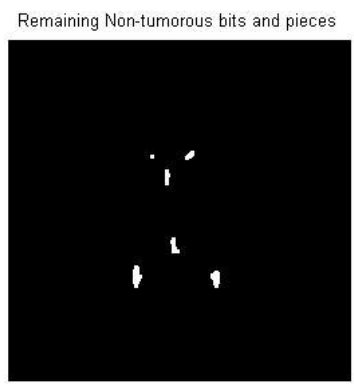

Figure 3: Remaining bits and pieces

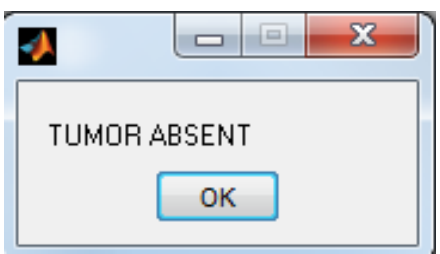

Figure 4: Tumor 'absent' decision dialog box

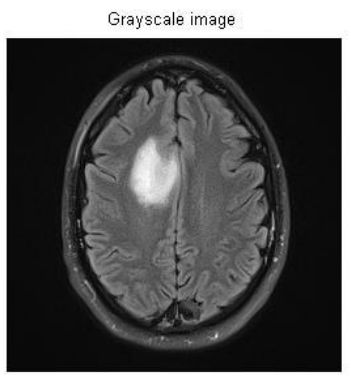

Figure 5: Original image

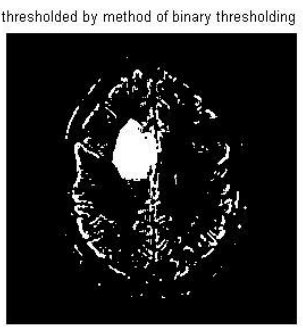

Figure 6: Thresholded image

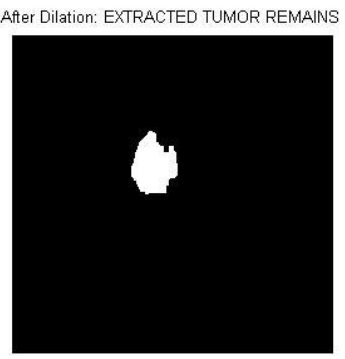

Figure 7: Extracted tumor 


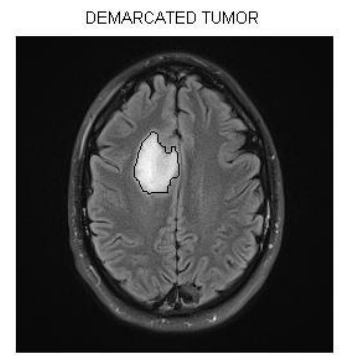

Figure 8: Demarcated Tumor

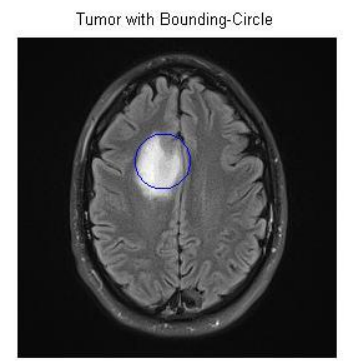

Figure 9: Tumor with bounding-circle

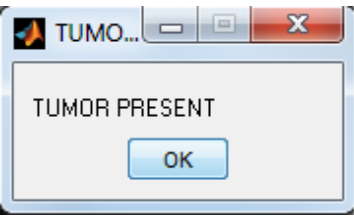

Figure 10: Tumor 'absent' decision dialog box

\section{CONCLUSION \& FUTURE SCOPE}

The technique proposed in this paper was successful in extracting the tumor portion; it has provided an accurate demarcation of the boundary of the tumor, along with correct visual location of the tumor with the help of a bounding circle. This work has also provided a diagnosis decision whether the tumor is present or absent along with the exact size of the tumor. This decision can assist as a supportive aid which can be used at the doctor's discretion in finally declaring a decision. A fuzzy clustering based segmentation of any brain MRI image has also been provided which can be used to study the intricate internal brain structure depending on the number of clusters chosen; hence it can be used as a study/analysis tool. The stage or grade of the tumor can also be calculated.

\section{REFERENCES}

[1] Gonzalez, R., Woods, R., 2009. Digital Image Processing, third ed. Pearson Education.

[2] Viner J. Brain Tumors. University of California, San Francisco Department of Neurosurgery. Available at http://nursing.ucsf medicalcenter.org/education/classMaterial/34_2.pdf. Accessed 17 May, 2013

[3] Dahab, D.A., Ghoniemy, S.A., Selim, G.M., 2012. Automated Brain Tumor Detection and Identification using Image Processing and Probabilistic Neural Network Techniques. International Journal of Image Processing and Visual Communication, 1-8
[4] Sinha, M., Mathur, K., 2012. Improved Brain Tumor Detection with Ontology. International Journal of Computational Engineering Research. 584-588

[5] Rajendran, A., Dhanasekaran, R. 2012. Brain Tumor Segmentation on MRI Brain Images with Fuzzy Clustering and GVF Snake Model. International Journal of Computation Communication. 530-539

[6] Noreen, N., Hayat, K., Madani, S., 2011. MRI Segmentation through Wavelets and Fuzzy C-Means. World Applied Sciences Journal 13 Special Issue of Applied Math).34-39

[7] Sajjad Mohsin, S., Sajjad, S., Malik, Z., Abdullah,A.H., 2012. Efficient Way of Skull Stripping in MRI to Detect Brain Tumor by Applying Morphological Operations, after Detection of False Background. International Journal of Information and Education Technology. 335337

[8] Mustaqeem, A., Javed, A., Fatima, T., 2012. An Efficient Brain Tumor Detection Algorithm using Watershed and Thresholding Based Segmentation. International Journal of Image, Graphics and Signal Processing. 34-39

[9] Vasuda, P., Satheesh S., 2010. Improved Fuzzy C-Means algorithm for MR brain image segmentation. International Journal on Computer Science and Engineering, 1713-1715

[10] Dasgupta, A., 2012. Demarcation of Brain Tumor using Modified Fuzzy C-Means. International Journal of Engineering Research and Applications. 529-533,

[11] Rakesh, M., Ravi, T., 2012. Image Segmentation and Detection of Tumor Objects in MR Brain Images Using Fuzzy C-Means(FCM) Algorithm. International Journal of Engineering Research and Applications. 2088-2094

[12] Soesanti, I., Susanto, A., Widodo, T.S., Tjokronagoro M., 2011. Optimized Fuzzy Logic Application for MRI Brain Images Segmentation. International Journal of Computer Science and Information Technology. 137-146

[13] Patil, R.C., Bhalchandra, A.S. Brain tumor extraction from MRI images using MATLAB. International Journal of Electronics, Communication \& Soft Computing Science and Engineering. 1-4

[14] Tonarelli, L., "Magnetic Resonance Imaging of Brain Tumor", Available at http://www.cewebsource.com/coursePDFs/MRIBrainTu mor

[15] Westbrook, C.,2010 MRI at a Glance, ISBN: 978-14051-4747-7, Wiley-Blackwell

[16] Tirpude, N.N., Welekar, R.R., 2013. Effect of Global Thresholding on Tumor-Bearing Brain MRI Images. International Journal of Engineering and Computer Science. 728-731

[17] Gonzalez, R., Woods R., 2008. Digital Image Processing, third ed. Pearson Education, Prentice Hall, pp. 741-742

[18] Gonzalez, R., Woods R., 2008. Digital Image Processing, third ed. Pearson Education, Prentice Hall, pp. 742-747

[19] Mohd Fauzi Othman, Mohd Ariffanan and Mohd Basri, "Probabilistic Neural Network for Brain Tumor Classification," 2nd International Conference on Intelligent Systems, Modelling and Simulation, 2011. 